\title{
Autoimmune hypoglycaemia caused by alpha-lipoic acid: a rare condition in Caucasian patients
}

\author{
A Veltroni', G Zambon', S Cingarlini² and M V Davì1 \\ ENETS Center of Excellence, 'Department of Medicine and 'Department of Oncology, University of Verona, \\ Verona, Italy
}

Correspondence should be addressed to A Veltroni

Email

alessio.veltroni@yahoo.com

\section{Summary}

Insulin autoimmune syndrome (IAS), a rare cause of autoimmune hyperinsulinaemic hypoglycaemia, is relatively well known in Japan. The incidence in Caucasians is less than one-fifth of that reported in Japanese people, but it is becoming increasingly recognised worldwide in non-Asians as well. Drugs containing sulphydryl groups are known to be associated with the disease in genetically predisposed individuals. Moreover, several recent reports showed a direct association between the onset of IAS and the consumption of dietary supplements containing alpha-lipoic acid (LA). Insulinoma remains the most prevalent cause of hypersulinaemic hypoglycaemia in Caucasians. Consequently, primary investigation in these patients is generally focused on localisation of the pancreatic tumour, often with invasive procedures followed by surgery. We described a case of an Italian woman presenting to us with severe recurrent hypoglycaemia associated with high insulin and C-peptide levels and no evidence of pancreatic lesions at imaging diagnostic procedures. She had taken LA until 2 weeks before hospitalisation. After an evaluation of her drug history, an autoimmune form of hypoglycaemia was suspected and the titre of insulin autoantibodies was found to be markedly elevated. This allowed us to diagnose LA-related IAS, thus preventing any unnecessary surgery and avoiding invasive diagnostic interventions.

\section{Learning points:}

- IAS is a rare cause of hyperinsulinaemic hypoglycaemia that typically affects Asian population, but it has been increasingly recognised in Caucasian patients.

- It should be considered among the differential diagnosis of hyperinsulinaemic hypoglycaemia to avoid unnecessary diagnostic investigations and surgery.

- It should be suspected in the presence of very high serum insulin levels (100-10 $000 \mu \mathrm{U} / \mathrm{mL})$ associated with high C-peptide levels.

- There is a strong association with administration of drugs containing sulphydryl groups included LA, a dietary supplement commonly used in Western countries to treat peripheral neuropathy.

\section{Background}

Insulin autoimmune syndrome (IAS), also known as Hirata's disease, is a rare autoimmune disorder characterised by hyperinsulinaemic hypoglycaemia associated with high titres of antibodies to endogenous insulin, in the absence of pathological abnormalities of the pancreatic islets and prior exposure to exogenous insulin (1). The syndrome is relatively well known in Japan, whereas it is considered rare in the rest of Asia, and it is extremely uncommon in Western countries. After the first case described in Japan in 1972 by Hirata and 
colleagues, there were 380 cases of IAS identified in Japan from 1970 to 2009 (2), where the syndrome is the third leading cause of hypoglycaemia following insulinoma and extrapancreatic neoplasms (3). Regarding non-Asian individuals, the incidence of the syndrome remains hard to estimate. A recent review of literature published in 2017 by Censi et al. has collected about 70 cases of IAS in Western Europe, North and South America (4), with a total of 12 Italian case reports. This confirms that the incidence in Europe, particularly in Italy, is extremely rare. In Caucasian patients, IAS affects men and women equally, and it is more common over 40 years of age while very rare in children (4).

IAS is characterised by episodes of hypoglycaemia occurring most often postprandially, with markedly elevated insulin levels, usually above $100-1000 \mu \mathrm{U} / \mathrm{mL}$. Such insulin serum levels are not usually seen in patients affected by insulinoma. C-peptide concentrations are typically increased or incompletely suppressed (3).

The exact mechanisms that cause IAS are not entirely clear. It is well known that there is a strong association with the HLA haplotype, and, in addition, it has been demonstrated that about half of the affected patients had recently been exposed to a sulphydryl-containing medication $(2,3)$, mainly methimazole and tiopronin. Two case reports have been associated with proton pump inhibitors (5). In such forms, IAS usually occurs from 4 to 6 weeks after the start of treatment (6).

Recently, an increasing number of cases have been associated with alpha-lipoic acid (LA) consumption (7). After the year 2000, LA gained popularity in Western countries as a dietary supplement with antioxidant and anti-inflammatory properties, especially for peripheral neuropathy in diabetic patients. The first case of LA-related IAS was described in Japan in 2003 (2), while the first Caucasian case was described in Italy in 2011 (8). Other secondary forms of IAS are associated with autoimmune diseases, haematological disorders, liver diseases and neoplasms (3).

\section{Case presentation}

A 56-year-old Caucasian, obese, non-diabetic woman was admitted to the emergency department for loss of consciousness that occurred in the late postprandial phase. On admission, very low plasma glucose levels were found (fingerstick blood sugar: $21 \mathrm{mg} / \mathrm{dL}$ ). She was treated with continuous infusions with $20 \%$ glucose and transferred to an Internal Medicine Department of an Italian peripheral hospital.
In the previous 2 weeks, the patient reported recurrent symptoms referring to hypoglycaemia (blurred vision, diaphoresis and confusion), mainly occurring $2-3 \mathrm{~h}$ after meal and resolving with food intake. Regarding the patient's past medical history, she had undergone a surgical transsphenoidal removal of a prolactin-secreting pituitary adenoma 20 years prior. She was healthy in the following years. One month before hospitalisation, she underwent a carpal tunnel decompression surgery, and then was recommended to take oral LA $600 \mathrm{mg}$ once a day as antioxidant agent to reduce neuropathy symptoms. She took the medication for 2 weeks and suspended it 15 days before the hospital admission, as she complained of asthenia and dizziness. At admission, she was not taking any other concurrent treatments containing sulphydryl groups.

\section{Investigation}

Her physical examination was unremarkable except for obesity (weight $98 \mathrm{~kg}$, BMI 35). During the first days of hospitalisation, she developed several spontaneous episodes of hypoglycaemia, both during fasting and postprandial phase. In some postprandial hypoglycaemic episodes, very high serum insulin levels $(>1000 \mu \mathrm{UI} / \mathrm{mL}$, up to $8500 \mu \mathrm{UI} / \mathrm{mL}$ ) and C-peptide (up to $18.4 \mathrm{ng} / \mathrm{mL}$ ) were observed. These data were confirmed by a fasting test that was suspended in the first $24 \mathrm{~h}$ due to symptomatic hypoglycaemia. Insulin levels were measured by electrochemiluminescence immunoassay by Roche Elecsys/E170 (Roche Diagnostics).

Her renal and liver function, thyroid profile, pituitary hormones and serum protein electrophoresis were in the normal range. Tests for general autoimmunity were negative.

During the hospital stay, she underwent a CT scan, an MRI of the abdomen and an endoscopic ultrasonography which did not reveal any pancreatic lesion. Given the negative imaging procedures and the persistent hypoglycaemic episodes, the patient was referred to our University Centre to complete the diagnostic investigation. We measured the levels of serum insulin during the hypoglycaemic episodes with a different insulin assay (ADVIA Centaur Chemiluminescent Technology), and they were confirmed to be high (up to $83 \mathrm{uUI} / \mathrm{mL}$ ), although they resulted significantly lower compared to the data determined at the first hospital. Given the high insulin levels, the recurrent hypoglycaemias, the recent consumption of LA and the negative localisation imaging, we measured insulin autoantibodies (IAA), which resulted 


\section{2-month follow-up}

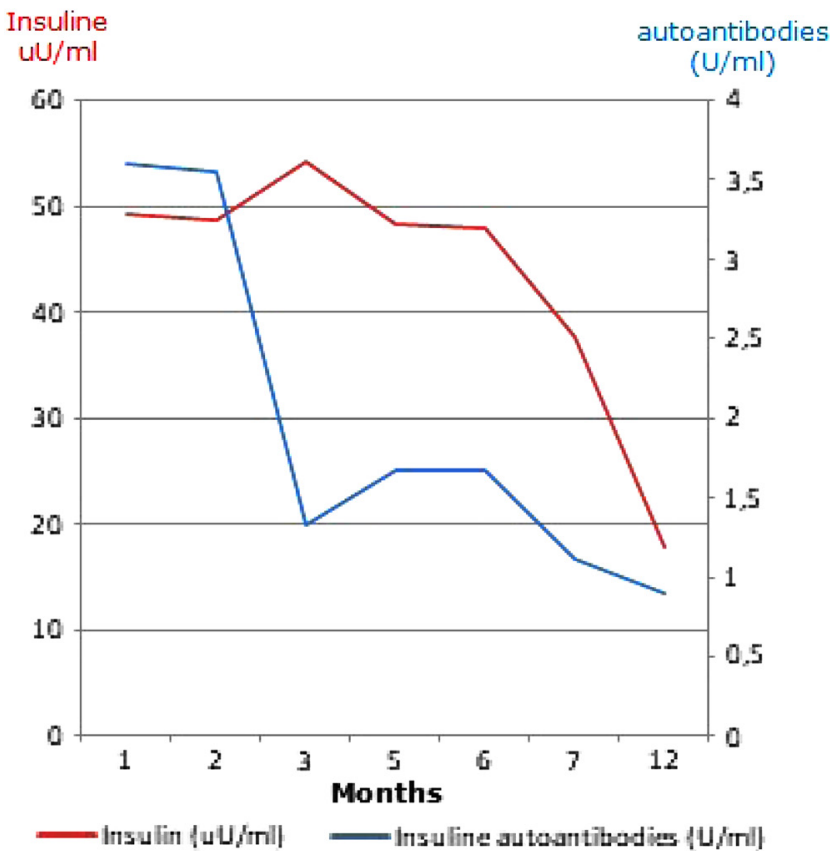

Figure 1

Twelve-month follow-up after the start of treatment.

positive in high titres (over 20U/mL, ELISA Medizym) confirming the diagnosis of IAS. HLA-II testing revealed the presence of HLA-DRB1*04:03.

\section{Treatment}

During the first week of hospitalisation in our centre, the patient required continuous glucose infusions. She was recommended to have small frequent meals, avoiding simple sugars and increasing complex carbohydrates to reduce postprandial hypoglycaemia.

Given the persistence of hypoglycaemic episodes along with the positivity of IAA, prednisone $50 \mathrm{mg}$ / day was started, obtaining a gradual decrease of insulin levels and an increase of fasting serum glycaemia in the first 2 weeks. She was consequently discharged from the hospital.

\section{Outcome and follow-up}

The steroid treatment was gradually tapered. After the beginning of therapy, serum glucose levels completely normalised after 1 month, IAA disappeared after 3 months and insulin values significantly decreased after 6 months. We stopped the treatment 6 months later and hypoglycaemia episodes were no longer detected. Insulin values spontaneously returned to normal range in 12 months (in particular, lab tests 6 months after the end of treatment were serum insulin $17.9 \mathrm{uU} / \mathrm{mL}$, C-peptide $0.64 \mathrm{ng} / \mathrm{mL}$, fasting glycaemia $81 \mathrm{mg} / \mathrm{dL}$ ). IAA remained negative in the following months. During this period, she continued a fractioned diet. She did not take any other supplement containing LA. (Fig. 1, Table 1).

\section{Discussion}

We described a rare case of LA-related IAS in a Caucasian woman. The diagnostic suspicion was based on the very high insulin levels, the negative imaging procedures for insulinoma, the hypoglycaemic episodes mainly in the postprandial phase, as well as her recent LA intake. The diagnosis was confirmed by the high titres of IAA. She was successfully treated with prednisone achieving a complete resolution of hypoglycaemia.

Regarding the causes of IAS, it is well known that, in approximately half of the patients the disease is related to exposure to drugs containing sulphydryl compounds (3). It has been proposed that these molecules may induce autoantibody formation by interacting with the disulphide bonds of the insulin molecule, making it more immunogenic (9). Also LA contains two sulphur atoms, which are reduced to two sulphydryl compounds stimulating the development of IAA (10).

Recent papers have shown a direct association between the appearance of IAS and LA intake in genetically predisposed people. A recent epidemiological analysis reported 18 cases of LA-related IAS in Japan from 2003 to 2012 and one case in Korea (10). To our knowledge, nine case studies of IAS caused by LA in Caucasians have been

Table 1 Trend of lab test over a 6-month therapy.

\begin{tabular}{l} 
\\
\hline Fasting Glycaemia (mg/dL) \\
Insulin $(\mu \mathrm{Ul} / \mathrm{mL})$ \\
C-peptide $(\mathrm{ng} / \mathrm{mL})$ \\
Insulin autoantibodies $(\mathrm{U} / \mathrm{mL})$
\end{tabular}

\begin{tabular}{c}
\hline Normal values \\
\hline $60-100$ \\
$4-24$ \\
$0.9-4$ \\
$<2.4$
\end{tabular}

\begin{tabular}{c}
\hline Basal tests \\
\hline 48 \\
82.9 \\
4.5 \\
$>20$ \\
\hline
\end{tabular}

\begin{tabular}{c}
\hline month of therapya \\
\hline 86 \\
49.3 \\
4.66 \\
3.6 \\
\hline
\end{tabular}

\begin{tabular}{c}
3 months of therapya \\
\hline 66 \\
51 \\
3.46 \\
1.33 \\
\hline
\end{tabular}

\begin{tabular}{c}
6 months of therapya \\
\hline 82 \\
47.9 \\
2.85 \\
1.67
\end{tabular}

aPrednisone dosage: $50 \mathrm{mg}$ daily for 3 months, then reduced by $5 \mathrm{mg}$ every 10 days and stopped 6 months after the beginning of treatment. 
published so far $(8,11,12,13)$. In all Caucasian patients, the treatment with LA was suspended on hospital admission, and the discontinuation of the drug resulted in a reduction of hypoglycaemic episodes. Our patient spontaneously interrupted the medication, but she experienced an episode of severe unconsciousness requiring an emergency treatment after 2 weeks of drug-free period. Therefore, it appears that the discontinuation of treatment does not always coincide with an immediate clinical resolution or a rapid improvement in laboratory tests.

The difference in serum insulin titres found in the two hospital laboratories may be related to a different crossreaction between autoantibodies binding the analyte and the immunoassay reagents in the two immunometric insulin assays. Free serum insulin levels should be estimated by PEG precipitation of IAA to separate free insulin from antibody-bound insulin; in cases of suspected IAS, a marked difference between the serum total insulin and free insulin is strongly suggestive of the disease (14).

A genetic predisposition also influences the occurrence of IAS, showing a strong correlation with certain HLA class II alleles, in particular HLA-DRB1*0406 in Japanese people and, to a lesser extent, HLA-DRB ${ }^{*} 04: 03$, especially in Caucasians (11). In our patient, HLA-II testing revealed the presence of HLA-DRB $1{ }^{*} 04: 03$, coherent with the majority of Caucasians reported in literature. The lower prevalence of IAS among Europeans can probably be explained by the lower prevalence of DRB1*04:06 in this population. However, the high occurrence of the DRB1*04:03 allele in Caucasians, combined with the growing use of LA in Western countries, may explain the increasing appearance of IAS in Europe.

Similar to most of the cases reported in literature, our patient presented mostly episodes of postprandial hypoglycaemia. The origin of this clinical feature is not completely clear. Most likely, the insulin molecules, secreted after a meal, bind their autoantibodies and, when glucose concentration falls in the bloodstream during the late postprandial phase, they spontaneously dissociate from the autoantibodies in an unregulated way, resulting in hypoglycaemia (3).

Despite the absence of an established treatment protocol, in the majority of patients IAS is a transient condition with spontaneous resolution within 3-6 months of diagnosis (15). Any precipitating drug must be discontinued. Small frequent meals poor in simple carbohydrates remain the first-line treatment of hypoglycaemia. Glucocorticoids may be useful as second-line therapy. Our patient had no resolution with dietary treatment, but had a satisfactory response only after the beginning of steroid treatment.

In conclusion, our case confirms that IAS should always be considered in the differential diagnosis of hyperinsulinaemic hypoglycaemia in non-diabetic individuals, especially in the presence of very high insulin levels, so as to avoid any unnecessary, invasive and costly procedures or surgery. An accurate drug history is essential. Despite the rarity of IAS, clinicians should be aware of the possible toxicity of nutritional supplements containing LA, bearing in mind the growing use of this compound nowadays.

\section{Declaration of interest}

The authors declare that there is no conflict of interest that could be perceived as prejudicing the impartiality of the research reported.

\section{Funding}

This work did not receive any specific grant from any funding agency in the public, commercial, or not-for-profit sector.

\section{Patient consent}

Written informed consent was obtained from the patient for publication of this article.

\section{Author contribution statement}

M V Davì and A Veltroni: diagnosed and followed up the case and wrote the paper. G Zambon and S Cingarlini worked on data collection.

\section{References}

1 Uchigata Y \& Hirata Y. Insulin autoimmune syndrome (IAS, Hirata disease). Annales de Medecine Interne 1999150 245-253.

2 Uchigata Y, Hirata Y \& Iwamoto Y. Drug-induced insulin autoimmune syndrome. Diabetes Research and Clinical Practice 2009 83 e19-e20. (https://doi.org/10.1016/j.diabres.2008.10.015)

3 Wong SL, Priestman A \& Holmes DT. Recurrent hypoglycemia from insulin autoimmune syndrome. Journal of General Internal Medicine 201429 250-254. (https://doi.org/10.1007/s11606-013-2588-9)

4 Censi S, Albergoni MP, Gallo N, Plebani M, Boscaro M \& Betterle C. Insulin autoimmune syndrome (Hirata's disease) in an Italian patient: a case report and review of the literature. Clinical Chemistry and Laboratory Medicine 201856 889-895. (https://doi.org/10.1515/ cclm-2017-0392)

5 Pooja S, Nitin T \& Abdulkadir O. Insulin autoimmune syndrome: a rare cause of postprandial hypoglycemia. Endocrinology, Diabetes and Metabolism Case Reports 2016 EDM16-0064. (https://doi.org/10.1530/ EDM-16-0064)

6 Balestrieri A, Magnani E, Ragazzini C \& Pasini G. Primary insulin autoimmune syndrome in an Italian woman: a case report. Italian Journal of Medicine 20159 169. (https://doi.org/10.4081/ itjm.2015.483) 
7 Furukawa N, Miyamura N, Nishida K, Motoshima H, Taketa K \& Araki E. Possible relevance of $\alpha$ lipoic acid contained in a health supplement in a case of insulin autoimmune syndrome. Diabetes Research and Clinical Practice 200775 366-367. (https://doi. org/10.1016/j.diabres.2006.07.005)

8 Bresciani E, Bussi A, Bazzigaluppi E \& Balestrieri G. Insulin autoimmune syndrome induced by $\alpha$-lipoic acid in a Caucasian woman: case report. Diabetes Care 201134 e146. (https://doi. org/10.2337/dc11-0600)

9 Redmon JB \& Nuttal FQ. Autoimmune hypoglycemia. Endocrinology and Metabolism Clinics of North America 199928 603-618, vii. (https://doi.org/10.1016/S0889-8529(05)70090-6)

10 Bae SM, Bae MN, Kim EY, Kim IK, Seo MW, Shin JK, Cho SR \& Jeong GH. Recurrent insulin autoimmune syndrome caused by alpha-lipoic acid in type 2 diabetes. Endocrinology and Metabolism 201328 326-330. (https://doi.org/10.3803/EnM.2013.28.4.326)

11 Gullo D, Evans JL, Sortino G, Goldfine ID \& Vigneri R. Insulin autoimmune syndrome (Hirata Disease) in European Caucasians taking $\alpha$-lipoic acid. Clinical Endocrinology 201481 204-209. (https:// doi.org/10.1111/cen.12334)

12 Corsi A, Cassibba S, Scaranna C, Dodesini AR, Lepore G \& Trevisan R. "Insulin autoimmune syndrome": due casi molto diversi. GIDM $2016 \mathbf{1 .}$

13 Ametrano AG, Losurdo F, Strippoli D, De Tullio A, Ippolito C, Laviola L, Bellomo Damato A \& Giorgino F. Un caso di ipoglicemia a digiuno e iperglicemia postprandiale da anticorpi anti-insulina. Giornale Italiano di Diabetologia e Metabolismo 201434 32-35.

14 Church D, Cardoso L, Bradbury S, Clarke C, Stears A, Dover A, Halsall D \& Semple R. Diagnosis of insulin autoimmune syndrome using polyethylene glycol precipitation and gel filtration chromatography with ex vivo insulin exchange. Clinical Endocrinology 201786 347-353. (https://doi.org/10.1111/cen.13179)

15 Paiva ES, Pereira AE, Lombardi MT, Nishida SK, Tachibanha TT, Ferrer C, Hauace OM, Vieira JG \& Reis AF. Insulin autoimmune syndrome (Hirata disease) as differential diagnosis in patients with hyperinsulinemic hypoglycemia. Pancreas 200632 431-432. (https:// doi.org/10.1097/01.mpa.0000220872.18153.72)

Received in final form 3 November 2018

Accepted 22 November 2018 